\title{
VALIDACIÓN DE ESCALA DE APOYO EN DUELO EN ATENCIÓN DE SALUD PARA POBLACIÓN HISPANOPARLANTE
}

\author{
SCALE VALIDATION OF GRIEF SUPPORT IN HEALTH CARE FOR SPANISH-SPEAKING \\ POPULATION
} \begin{abstract}
González ${ }^{1}$
' Escuela de Enfermería, Pontificia Universidad Católica de Chile, Santiago, Chile.

2 Subdirección de Operaciones, Instituto Nacional de Estadísticas de Chile.

${ }^{3}$ Servicio de Oncología, Clínica Universidad Católica- Christus Health, Chile.
\end{abstract}

Paula Vega Vega', José Bustos Melo², Rina González Rodríguez', Natalie Santibáñez Galdames ${ }^{3}$, Antonia Sateler Villanueva', Valentina Cortínez Rojas ${ }^{7}$ y María José Viedma

Resumen

La muerte de un niño atendido por profesionales puede ser concebida por ellos como una pérdida, convirtiéndose en ocasiones en una experiencia traumática. El soporte social se transforma en un importante mediador del proceso de duelo, ayudando a aliviar el estrés y afrontarlo de mejor manera. Objetivo: Traducción y validación al español del Grief Support in Health Care Scale (GSHCS). Método: Tras la traducción y validación de la equivalencia transcultural del GSHCS en su versión al español: Escala de Apoyo en Duelo en Atención de Salud (EADAS), se procedió a la auto aplicación del instrumento en 215 profesionales que trabajan en unidades de oncología y de cuidados críticos pediátricos. Con los datos se realizó un análisis de consistencia interna, Alfa de Cronbach, intervalos de confianza de Bootstrap y análisis exploratorio para testear las propiedades psicométricas de la escala dividida en tres dimensiones: reconocimiento de la relación, reconocimiento de la pérdida e incorporación de los rituales, realizándose una comparación de medias mediante la prueba de t para muestras independientes. Resultados: El EADAS obtuvo una validación y confiabilidad con un Coeficiente Alfa de Cronbach de 0,81. Al analizar las variables, se encontró que los
Abstract

The death of a child, who is attended by professionals, can be conceived by them as a loss, sometimes resulting in a traumatic experience. Social support becomes an important mediator of the grieving process, helping to relieve the stress and to cope better. Objective: Translation and validation into Spanish of "Grief Support in Health Care Scale (GS$H C S)$ ». Method: Through the translation and validation of cross-cultural equivalence of GSHCS in its Spanish version: "Apoyo en Duelo en Atención de Salud (EADAS)», we proceeded to auto-apply this instrument to 215 professionals working in oncology units and critical pediatric care. With this data there was an internal analysis of the tendencies using Cronbach's Alpha, Bootstrap Confidence Intervals and an exploratory analysis was performed to test the psychometric properties of the scale divided into three dimensions; recognition of the relationship, recognition of the loss and incorporation of belief rituals, performing a comparison of means using the $t$ test for independent samples. Results: The "Grief Support in Health Care Scale» (GSHCS) obtained a validation and reliability with a coefficient Cronbach's Alpha score of 0.81. By analyzing the variables, it was found that oncology unit

\section{Correspondencia:}

Paula Vega. Vicuña Mackenna 4860,

Macúl Santiago, Chile.

E-mail: pvegav@uc.cl
Proyecto financiado por el Fondo PUENTE de la Vicerrectoría de Investigación de la Pontificia Universidad Católica de Chile. $N^{\circ}$ 9/2014. La organización que promocionó el financiamiento no tuvo influencia en el diseño del estudio; en la relación, análisis o interpretación de los datos; en la preparación, revisión o aprobación del manuscrito. 
profesionales de la unidad de oncología y las mujeres de ambas unidades se perciben más socialmente apoyados en sus duelos. Conclusión: El EADAS es un instrumento válido y confiable que permite identificar la percepción de soporte ante el duelo en profesionales de la salud.

Palabras claves: Duelo, profesionales de la salud, soporte social. professionals and both units women perceive themselves to be more socially supported in their mourning. Conclusion: The "Grief Support in Health Care Scale» (GSHCS) is a valid and reliable instrument, which allows to identify the perception of support in the mourning process.

Keywords: Grief, Health professional, social support.

\section{INTRODUCCIÓN}

La medicina ha experimentado avances que han permitido extender la vida, y con ello generar un cambio de paradigma frente a la muerte ${ }^{(1,2)}$, atribuyéndole una connotación negativa ${ }^{(3)}$. Para los equipos de salud que atienden niños con enfermedades crónicas les es difícil enfrentar la muerte, por lo incomprensible que puede llegar a ser el fallecimiento en la infancia ${ }^{(4)}$. Este evento puede aquejarlos, siendo concebido como una pérdida, especialmente cuando se han generado vínculos afectivos ${ }^{(5,6)}$, convirtiéndose en una situación traumática para los profesionales $^{(7)}$.

Los duelos en ellos han sido vistos con prejuicio, dada la percepción de que están inhabilitados para vivenciar la muerte de los pacientes como una pérdida propia ${ }^{(5)}$. La teoría de "Privación al Derecho de Luto" de Doka ${ }^{(8)}$, da cuenta que en la sociedad existen reglas que determinan quien puede o no estar en duelo, y con ello, cuales son las respuestas apropiadas de pérdidas. Esta teoría propone que los proveedores de salud recibirían soporte necesario cuando la relación con sus pacientes fuese concebida como real por los otros, validando de esa manera su duelo, y así ser incluidos en los rituales funerarios. Sin embargo, aquellos que les faltó recibir estos apoyos, se les generó dificultades para afrontar la muerte de sus pacientes y desarrollaron duelos complicados ${ }^{(8,9)}$.
Algunas investigaciones han demostrado que el soporte social dentro del equipo de salud es un importante mediador de este proceso, ayudando a aliviar el estrés y permitiendo afrontar adecuadamente las pérdidas de los pacientes ${ }^{(10-12)}$.

Existe un instrumento psicométrico: "Grief Support in Health Care Scale" (GSHCS), que permite evaluar la percepción de soporte social de los profesionales de salud, elaborado por el Dr. Keith Anderson et al. (2010)(13), el cual está validado y tiene coeficientes que sustentan su confiabilidad. El coeficiente Alfa de Cronbach para la dimensión 1 (Subescala de reconocimiento de la relación), dimensión 2 (Subescala de reconocimiento de la pérdida) y dimensión 3 (Subescala de inclusión en los ritos funerarios), fueron $0,89,0,86$, y 0,78 , respectivamente $^{(13)}$. Sin embargo se desconoce su confiabilidad en población hispana.

Así el propósito del presente estudio es validar la versión en español para contar con un instrumento que permita medir el soporte social percibido por los profesionales de la salud hispanoparlantes, frente a sus duelos.

\section{MATERIAL Y MÉTODO}

El estudio de validación para el instrumento GSHCS al idioma español, fue realizado el 2014 en una muestra por conveniencia de trabajadores de cuatro centros de salud del Sistema Público de Santiago 
de Chile. Para el logro de los objetivos, el proceso se desarrolló en tres fases: Traducción del instrumento a evaluar, considerando la traducción inversa, la adaptación cultural y de lenguaje; Aplicación de la escala GSHCS en español a los participantes y Estudio de concordancia, confiabilidad y validez. Se invitó a participar a todos los profesionales médicos, becados, enfermeros, psicólogos, terapeutas ocupacionales y técnicos de salud, que trabajaban en las unidades de hospitalización, policlínicos de oncología (ONCO) y de cuidados intensivos ( $\mathrm{UCl}$ ) de los centros seleccionados. Los criterios de inclusión fueron: trabajar en una de las unidades al momento del estudio por más de 6 meses, percibirse como deudo de sus pacientes y aceptar participar voluntariamente bajo consentimiento informado.

Este estudio contó con la aprobación del Comité Ético Científico de la Facultad de Medicina de la Pontificia Universidad Católica de Chile y los Comités de Ética de cada uno de los Servicios de Salud involucrados.

\section{Instrumento}

La escala, desarrollada por Anderson, Ewen y Miles (2010) ${ }^{(13)}$, consideró los componentes de la Teoría de la Privación al Derecho al Luto $^{(8)}$ : Reconocimiento de la relación, Reconocimiento de la pérdida e Inclusión en los rituales funerarios.

Esta escala está formada por 15 ítems. Los primeros cinco son usados para sondear el grado en que es reconocida la relación con sus pacientes por su propia familia, amigos, compañeros de trabajo, directivos, y familiares de los pacientes (Subescala 1). Los segundos cinco, sondean el grado en el cual el duelo que vivencian es reconocido por los grupos anteriormente mencionados (Subescala 2). Los últimos cinco, miden la inclusión de los profesionales en los servicios funerarios y rituales de sus pacien- tes (Subescala 3). Para medir el nivel de acuerdo a desacuerdo en estos ítems, se usa una escala de Likert de 5 puntos. Un alto puntaje, indica alto nivel de percepción de soporte social ante el duelo, y un puntaje bajo, una percepción deficiente.

\section{Procedimiento}

Traducción y validación cultural de la escala

En la primera fase se realizó la traducción y validación de la equivalencia transcultural del instrumento GSHCS en su versión del inglés al español, de manera de lograr una correspondencia de: contenido, semántica, técnica, criterio y conceptual, entre la versión original y la versión en español ${ }^{(14,15)}$. El proceso comenzó con la traducción de la escala GSHCS al español, por una enfermera bilingüe con entrenamiento en la temática de duelo, cuya lengua materna era el español. Una vez traducida, esta versión fue analizada por tres profesionales bilingües, dos enfermeras y una psicooncóloga, expertas en soporte social y duelo. Tras verificar la coherencia entre los conceptos y cumplimiento de la equivalencia transcultural entre ambas versiones, se gestó una segunda versión en español, la cual fue sometida a confirmación ${ }^{(16)}$. Para ello se presentó el instrumento a 15 profesionales de la salud de otras áreas de la pediatría, quienes evaluaron la comprensión de los ítems y el tiempo de respuesta, desarrollándose la versión definitiva. Esta tercera versión en español, fue sometida a una traducción inversa ${ }^{(17)}$ al idioma inglés por una traductora bilingüe, cuya lengua materna era el inglés, con el objeto de verificar la coherencia entre la versión original del instrumento y la versión adaptada. Ambas fueron enviadas al autor del instrumento, quien verificó la coherencia con la versión original y autorizó su aplicación para el presente estudio. Se obtuvo así la versión en español: "Escala de Apoyo en 
Duelo en Atención de Salud-EADAS" (Anexo 1), el cual mantiene el formato original.

Para la segunda fase se invitó a todos los profesionales y técnicos de salud que cumplían los criterios de inclusión, dado que ellos fueron quienes estuvieron expuestos a la vivencia de duelo al experimentar la muerte de los niños/adolescentes que atendieron en las unidades elegidas. En cada centro, un asistente de terreno entrenado contactó a los interesados por hospital. Tras leer y firmar ambos el consentimiento, se realizó la auto aplicación de la encuesta EADAS y un cuestionario sobre aspectos sociodemográficos.

\section{Análisis}

Los datos fueron digitados en una planilla electrónica. Posteriormente se usaron controles de calidad de estos, para asegurar la exactitud en la digitación. A su vez, se realizó un análisis para datos perdidos, identificando que menos del $3 \%$ de cada ítem del instrumento tenía datos perdidos. Luego, los datos perdidos fueron imputados usando estimación de máxima verosimilitud $^{(18)}$. Inicialmente, se realizó un Análisis Factorial Exploratorio(19) en los ítems del instrumento en un total de 215 cuestionarios, a fin de verificar las relaciones entre las escalas de los ítems y la identificación de los factores. La consistencia interna de estos fue estimada mediante el coeficiente de Alfa de Cronbach ${ }^{(20)}$. Luego se estimó el intervalo de confianza Bootstrap percentil del 95\% (ICBP95\%), para el que fueron realizados 1000 re-muestreos en cada estimación ${ }^{(21)}$.

Además, se analizaron las diferencias en relación al género, estado marital, lugar de trabajo (ONCO versus $\mathrm{UCl}$ ), años laborales sobre las 3 subescalas de la EADAS, a través de comparaciones de medias mediante la prueba de t para muestras independientes, previa verificación de supuestos.
Los análisis estadísticos fueron realizados considerando un nivel de significación del $5 \%$, utilizando como soporte el software estadístico y gráfico $R^{(22)}$, junto a la librería "psy" de Falissard ${ }^{(20)}$.

\section{RESULTADOS}

\section{Caracterización de la muestra}

Para el estudio de validación de la EADAS se reclutó una muestra de 215 trabajadores de la salud. El estudio mostró que: $84,7 \%$ eran mujeres, $50,7 \%$ procedían de Unidades de Oncología; 59,5\% correspondió a trabajadores con título profesional en el área. En la tabla 1 se presentan las características socio-demográficas registradas. Los puntajes tuvieron una completitud del $94,3 \%$, por tanto ese complemento fue estimado mediante la Estimación Máxima Verosimilitud. Con la prueba de normalidad de Shapiro Wilks se pudo concluir que las subescalas en estudio presentaron distribución normal. Asi mismo, la distribución del puntaje total de la prueba, fue normal.

\section{Análisis de EADAS}

Tras el análisis de cada subescala según sus ítemes, fue posible estimar la media y desviación estándar. Para determinar la confiabilidad -como consistencia internase utilizó el método Alfa de Cronbach, cuyo índice para el total de 215 cuestionarios realizados, alcanzó a 0,81, indicando alta confiabilidad de la escala total. Al calcular el coeficiente Alfa de Cronbach para cada subescala planteada teóricamente en la adaptación del test, se obtuvieron valores de $0,74,0,81$ y 0,70 , para la dimensión 1,2 y 3 , respectivamente. Siendo la subescala 3 (Inclusión a rituales funerarios) la que mostró la menor consistencia interna, pero con un valor considerado bueno. Para confirmar estas dimensiones, se realizó un 
Tabla 1. Característica de la muestra

\begin{tabular}{|c|c|c|}
\hline & $\mathbf{n}$ & Frecuencia (\%) \\
\hline \multicolumn{3}{|l|}{ Sexo } \\
\hline $\mathrm{F}$ & 182 & 84,7 \\
\hline M & 33 & 15,4 \\
\hline \multicolumn{3}{|l|}{ Estado civil } \\
\hline Soltero/a & 78 & 36,3 \\
\hline Casado/a & 137 & 63,7 \\
\hline \multicolumn{3}{|l|}{ Nivel de estudios } \\
\hline Educación Básica & 2 & 0,94 \\
\hline Educación Media & 14 & 6,51 \\
\hline Profesional & 135 & 62,79 \\
\hline Técnico & 64 & 29,76 \\
\hline \multicolumn{3}{|l|}{ Título profesional } \\
\hline Auxiliar & 11 & 5,11 \\
\hline Profesional & 128 & 59,53 \\
\hline Técnico & 76 & 35,34 \\
\hline \multicolumn{3}{|l|}{ Tiempo de ejercicio profesional } \\
\hline Menos de 1 año & 29 & 13,5 \\
\hline 1 a 5 años & 74 & 34,4 \\
\hline 6 a 10 años & 35 & 16,3 \\
\hline 11 a 15 años & 19 & 8,8 \\
\hline Más de 15 años & 58 & 27,0 \\
\hline \multicolumn{3}{|l|}{ Unidad de desempeño } \\
\hline Oncología & 109 & 50,7 \\
\hline Unidad de Cuidados Intensivos & 106 & 49,3 \\
\hline \multicolumn{3}{|l|}{ Participación en cursos de duelo } \\
\hline no & 154 & 71,6 \\
\hline si & 61 & 28,4 \\
\hline Total & 215 & 100 \\
\hline
\end{tabular}

Análisis Factorial Exploratorio de Máxima Verisimilitud con rotación varimax para poner a prueba 3 factores, el que resultó con un Chi cuadrado de 272,36 con 63 grados de libertad y un valor $\mathrm{p}$ menor al 0,05. Según la Tabla 2, la pregunta 6, 7, 8 y 10 están correlacionadas con el Factor 1 que corresponde a "reconocimiento del sufrimiento del profesional frente a la muerte del paciente por otros"; la pregunta 11,12 y 13 corresponderían al Factor 2 , el cual refleja la "incorporación de los profesionales a los rituales funerarios de los pacientes" y las preguntas 3, 4 y 9, que correspondería al Factor 3, la cual refleja "el reconocimiento de la cercanía del profesional a sus pacientes".
En la tabla 3 es posible confirmar el resumen de las estadísticas descriptivas y los valores del coeficiente Alfa de Cronbach total y para cada una de las 3 subescalas. Se estimó el Intervalo de Confianza Bootstrap Percentil del 95\% (ICBP 95\%) para cada una de ellas. Así fue posible identificar la media del puntaje total de las tres subescalas con 50,02 y una desviación estándar (DS) de 8,48. Estos valores del coeficiente de consistencia, evidencian que la encuesta obtiene la información de forma fidedigna y por tanto, resulta consistente para medir el duelo en profesionales de la salud.

En la tabla 4 se presentan las correlaciones entre las puntuaciones de los distintos ítems de la EADAS. Se observa que la sub- 
Tabla 2. Cargas de los Factores del Análisis Factorial Exploratorio

\begin{tabular}{|l|c|c|c|}
\multicolumn{5}{c|}{ Factor1 } & Factor2 & Factor3 \\
\hline Preg6 & 0,75 & & \\
Preg7 & 0,76 & & \\
Preg8 & 0,59 & & \\
Preg10 & 0,66 & & \\
Preg11 & & 0,73 & \\
Preg12 & & 0,88 & \\
Preg13 & 0,30 & 0,58 & \\
Preg3 & & & 0,53 \\
Preg4 & 0,38 & & 0,99 \\
Preg9 & 0,49 & & \\
Preg1 & 0,41 & & \\
Preg2 & 0,39 & & \\
Preg5 & & 0,38 & \\
Preg14 & & & \\
Preg15 & & & \\
\hline
\end{tabular}

\begin{tabular}{|l|c|c|c|}
\hline & Factor1 & Factor2 & Factor3 \\
\hline SS loadings & 2,88 & 2,01 & 1,87 \\
\hline Proportion Var & 0,19 & 0,13 & 0,12 \\
\hline Cumulative Var & 0,19 & 0,33 & 0,45 \\
\hline
\end{tabular}

Para la prueba de hipótesis, los 3 factores son suficientes, con un chi cuadrado es de 272,36 con 63 grados de libertad y un valor $\mathrm{p}$ menor al 0,05.

\section{Tabla 3. Resumen de estadísticas del análisis de la EADAS por dimensión}

\begin{tabular}{|lccccc|}
\hline \multicolumn{1}{|c}{ Factor } & Ítems & Media & DS & $\alpha$ de Crombach & ICBP95\% \\
\hline Las tres dimensiones & 15 & 50,02 & 8,48 & 0,81 & {$[0,77-0,85]$} \\
\hline Reconocimiento de la Relación & 1 al 5 & 20,42 & 3,24 & 0,74 & {$[0,67-0,80]$} \\
\hline Reconocimiento de la Pérdida & 6 al 10 & 18,67 & 3,84 & 0,81 & {$[0,75-0,85]$} \\
\hline Inclusión en ritual funerario & 11 al 15 & 10,93 & 4,25 & 0,70 & {$[0,63-0,76]$} \\
\hline
\end{tabular}

EADAS=Escala de apoyo en duelo en atención de salud

DS= Desviación Estándar, $\alpha$ de Crombach = Coeficiente de Alfa de Crombach, ICBP95\%= Intervalo de confianza Boostrap percentil del 95\%.

escala 1 (ítem 1 al 5), presenta mayor frecuencia de correlaciones significativas con la subescala 3 (ítem 11 al 15), y en menor grado con la subescala 2 (ítem 6 al 10). Especial relevancia toma el ítem 13 y 14, los que tienen una mayor frecuencia de correlaciones significativas con el total de los ítems. Por lo que se puede inferir, que al sentirse los trabajadores de la salud reconocidos desde su cercanía y pérdida con sus pacientes, 
a menudo pueden ser invitados y participan en los rituales funerarios. Al realizar el análisis de correlaciones de cada ítem con el total de la escala, se observan los ítems que más consistencia aportan a la escala (tabla 5).

Tras analizar las correlaciones entre cada ítem y la escala total, se evidencia que la subescala 1: "Reconocimiento de la relación con sus pacientes" muestra una alta consistencia interna, siendo el ítem 5 el que menor aporta (con una correlación de 0,6$)$. En el caso de la subescala 2: "Reconocimiento del sufrimiento" todos los ítems agregan información, siendo la subescala que más aportó al instrumento. Por otro lado, la subescala 3: "Inclusión en rituales funerarios", el ítem 11 y 15 son los que menos información entregan, con un 0,6 de correlación con la subescala.

\section{Hallazgos}

Para evaluar eventuales diferencias entre distintas características de los trabajadores encuestados, se realizaron comparaciones de los puntajes promedios totales y de las subescalas entre grupos, para los distintos grupos identificados. No se encontraron diferencias en relación al estado marital, años de desempeño profesional y entre estamentos. Sin embargo, se encontró que el género y la unidad de desempeño laboral mostraron diferencias estadísticamente significativas. En cuanto al género, las mujeres mostraron valores levemente mayores que los hombres $(\mathrm{t}=2,1389$, df $=32,689$, valor-p $<0,05)$. Esto está dado por la diferencia significativa en la subescala 1 ( $\mathrm{t}=2,6981, \mathrm{gl}=35,932$, valor-p $<$ $0,05)$, no observándose en la subescala 2 $(\mathrm{t}=1,595, \mathrm{gl}=34,466$, valor-p $>0,05) \mathrm{y}$ en la subescala $3(t=1,0283, g l=37,519$, valor-p > 0,05) (figura 1). Respecto a la unidad de desempeño laboral entre trabajadores de las unidades de ONCO y UCI, se observó diferencias significativas $(\mathrm{t}=3,7$, $\mathrm{gl}=203,634$, valor-p $<0,05)$ en cuanto a los puntajes totales. Esto se debió a la diferencia significativa en la subescala 1 ( $\mathrm{t}=$ $2,1407, \mathrm{gl}=203,131$, valor-p $<0,05$ ) y en la subescala $3(t=4,2297, g \mid=203,506$, valor-p $<0,05)$. No se presentaron diferencias en cuanto a la subescala $2(t=1,4925$, $\mathrm{gl}=201,066$, valor-p $>0,05$ ) (figura 2).

\section{Tabla 4. Correlaciones entre los distintos ítems de la EADAS (rs)}

\begin{tabular}{|c|c|c|c|c|c|c|c|c|c|c|c|c|c|c|c|}
\hline & Item1 & Item2 & Item3 & Item4 & Item5 & Item6 & Item7 & Item8 & Item9 & Item10 & Item11 & Item12 & Item13 & Item14 & Item15 \\
\hline Item1 & $\mathbf{1}$ & 0,59 & 0,33 & 0,28 & 0,37 & 0,45 & 0,33 & 0,24 & $0,22^{*}$ & 0,3 & $-0,04$ & 0,08 & $0,19^{*}$ & $0,19^{*}$ & $0,18^{*}$ \\
Item2 & - & $\mathbf{1}$ & 0,35 & 0,32 & 0,41 & 0,28 & 0,39 & 0,24 & $0,22^{*}$ & $0,26^{*}$ & 0,09 & $0,16^{*}$ & $0,19^{*}$ & $0,19^{*}$ & $0,14^{*}$ \\
Item3 & - & - & $\mathbf{1}$ & 0,54 & $0,24^{*}$ & $0,24^{*}$ & 0,27 & 0,43 & 0,33 & $0,16^{*}$ & $-0,08$ & 0,01 & $0,15^{*}$ & $0,16^{*}$ & $0,21^{*}$ \\
Item4 & - & - & - & $\mathbf{1}$ & 0,31 & 0,12 & $0,18^{*}$ & $0,23^{*}$ & 0,56 & 0,13 & 0,05 & 0,09 & $0,14^{*}$ & $0,17^{*}$ & 0,1 \\
Item5 & - & - & - & - & $\mathbf{1}$ & 0,28 & $0,25^{*}$ & $0,26^{*}$ & $0,24^{*}$ & 0,43 & 0,03 & $0,14^{*}$ & $0,26^{*}$ & $0,24^{*}$ & $0,22^{*}$ \\
Item6 & - & - & - & - & - & $\mathbf{1}$ & 0,68 & 0,42 & 0,27 & 0,46 & $-0,07$ & 0,05 & 0,11 & $0,18^{*}$ & 0,13 \\
Item7 & - & - & - & - & - & - & $\mathbf{1}$ & 0,52 & 0,38 & 0,48 & $-0,02$ & 0,11 & $0,18^{*}$ & $0,19 *$ & 0,1 \\
Item8 & - & - & - & - & - & - & - & $\mathbf{1}$ & 0,51 & 0,41 & $-0,06$ & 0,08 & $0,19 *$ & 0,15 & 0,04 \\
Item9 & - & - & - & - & - & - & - & - & $\mathbf{1}$ & 0,46 & 0,12 & $0,14 *$ & 0,29 & $0,22 *$ & 0,07 \\
Item10 & - & - & - & - & - & - & - & - & - & $\mathbf{1}$ & $-0,07$ & 0,04 & 0,32 & 0,29 & 0,07 \\
Item11 & - & - & - & - & - & - & - & - & - & - & $\mathbf{1}$ & 0,66 & 0,38 & 0,13 & 0,11 \\
Item12 & - & - & - & - & - & - & - & - & - & - & - & $\mathbf{1}$ & 0,49 & 0,34 & $0,24 *$ \\
Item13 & - & - & - & - & - & - & - & - & - & - & - & - & $\mathbf{1}$ & 0,47 & 0,27 \\
Item14 & - & - & - & - & - & - & - & - & - & - & - & - & - & $\mathbf{1}$ & 0,27 \\
Item15 & - & - & - & - & - & - & - & - & - & - & - & - & - & - & $\mathbf{1}$ \\
\hline
\end{tabular}

Los * indican correlaciones significativas. 
Tabla 5. Correlaciones de cada pregunta con el total de la escala y sus dimensiones.

Correlación: Dimensión 1 con las preguntas que la componen

\begin{tabular}{lllllll} 
& Preg1 & Preg2 & Preg3 & Preg4 & Preg5 & d1 \\
\hline Preg1 & 1,0 & 0,6 & 0,3 & 0,3 & 0,4 & 0,7 \\
Preg2 & 0,6 & 1,0 & 0,3 & 0,3 & 0,4 & 0,8 \\
Preg3 & 0,3 & 0,3 & 1,0 & 0,5 & 0,2 & 0,7 \\
Preg4 & 0,3 & 0,3 & 0,5 & 1,0 & 0,3 & 0,7 \\
Preg5 & 0,4 & 0,4 & 0,2 & 0,3 & 1,0 & 0,6 \\
d1 & 0,7 & 0,8 & 0,7 & 0,7 & 0,6 & 1,0 \\
\hline
\end{tabular}

Correlación: Dimensión 2 con las preguntas que la componen

\begin{tabular}{lllllll} 
& Preg6 & Preg7 & Preg8 & Preg9 & Preg10 & d2 \\
\hline Preg6 & 1,0 & 0,7 & 0,4 & 0,3 & 0,5 & 0,8 \\
Preg7 & 0,7 & 1,0 & 0,5 & 0,4 & 0,5 & 0,8 \\
Preg8 & 0,4 & 0,5 & 1,0 & 0,5 & 0,4 & 0,7 \\
Preg9 & 0,3 & 0,4 & 0,5 & 1,0 & 0,5 & 0,7 \\
Preg10 & 0,5 & 0,5 & 0,4 & 0,5 & 1,0 & 0,7 \\
d2 & 0,8 & 0,8 & 0,7 & 0,7 & 0,7 & 1,0 \\
\hline
\end{tabular}

Correlación: Dimensión 3 con las preguntas que la componen

\begin{tabular}{lllllll} 
& Preg11 & Preg12 & Preg13 & Preg14 & Preg15 & d3 \\
\hline Preg11 & 1,0 & 0,7 & 0,4 & 0,1 & 0,1 & 0,6 \\
Preg12 & 0,7 & 1,0 & 0,5 & 0,3 & 0,2 & 0,8 \\
Preg13 & 0,4 & 0,5 & 1,0 & 0,5 & 0,3 & 0,8 \\
Preg14 & 0,1 & 0,3 & 0,5 & 1,0 & 0,3 & 0,7 \\
Preg15 & 0,1 & 0,2 & 0,3 & 0,3 & 1,0 & 0,6 \\
d3 & 0,6 & 0,8 & 0,8 & 0,7 & 0,6 & 1,0 \\
\hline
\end{tabular}

Figura 1. Relación género y las dimensiones del GSHCSes
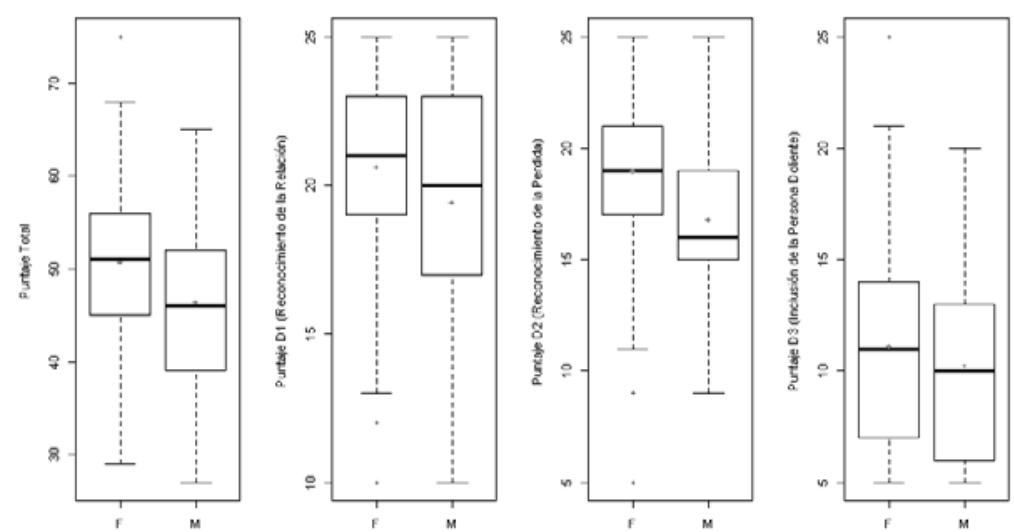

Puntajes totales y por dimensión en función al género ( $F=$ femenino y $M=$ masculino) en el total de 2015 trabajadores encuestados. Los símbolos $\left(^{*}\right)$ sobre las figuras indican diferencias estadísticamente significativas 
Figura 2. Relación unidad de desempeño laboral y las dimensiones del GSHCSes


Puntajes totales y por dimensión en función a la unidad de desempeño $(\mathrm{ONCO}=$ Oncología y UCl= Unidad Cuidados Intensivos) en el total de 2015 trabajadores encuestados. Los símbolos $\left({ }^{*}\right)$ sobre las figuras indican diferencias estadísticamente significativas.

\section{DISCUSIÓN}

La Escala de Apoyo en Duelo en Atención de Salud es un instrumento de medición psicométrica para cuantificar la percepción de soporte social ante el duelo en trabajadores de la salud. En esta investigación se pudo determinar la equivalencia transcultural considerando: contenido, semántica, técnica, criterio y aspecto conceptual, observándose que el instrumento traducido al español tuvo una buena comprensión por parte de los participantes, que se reflejó en un tiempo de llenado menor a 7 minutos, facilitando su auto aplicación.

Respecto a la validación psicométrica, los datos sugieren que la EADAS es confiable y tiene la validez para medir la percepción de apoyo ante el duelo de los profesionales de la salud hispanoparlantes, siendo capaz de cuantificar el reconocimiento y autentificación de la relación entre el trabajador de salud y la persona bajo su cuidado, el reconocimiento de las pérdidas por parte de los familiares, compañeros y deudos, y la posibilidad de ser incorporados en rituales funerarios de sus pacientes.

Según Oviedo et al. ${ }^{(23)}$ el valor mínimo aceptable para el coeficiente Alfa de Cronbach es 0,70. En este estudio se al- canzó 0,81 ; por lo que se puede afirmar que la EADAS posee consistencia interna sin redundancia, debido a que todos los valores estimados para las 3 subescalas, estuvieron en rangos aceptables sobre 0,70 y menor de 0,90. De la primera a la tercera subescala del instrumento, los valores oscilaron entre 0,70 y 0,81 , siendo aceptables en su consistencia interna. En el caso particular de la tercera subescala se repite el patrón observado en la validación del GSHCS por Anderson et al. ${ }^{(13)}$. En el caso del EADAS, estos valores son levemente inferiores a los de la escala original, lo que puede deberse a características socioculturales particulares de la población chilena y/o a una menor muestra utilizada(13).

Por otra parte, la matriz de correlaciones mostró que los profesionales se sienten apoyados en la incorporación a los rituales funerarios porque socialmente se reconoce su relación con los pacientes, más que el reconocimiento de la pérdida, lo que confirmaría lo planteado por Doka ${ }^{(8)}$.

En relación a los resultados observados tras el análisis de los puntajes del instrumento, las mujeres obtuvieron valores levemente mayores que los hombres, asi los datos sugieren que ellas tendrían una mayor percepción de apoyo social frente 
a sus duelos. Según algunos autores ${ }^{(4,24)}$, esto se puede dar por la mayor intensidad de reacciones que presentan los hombres frente a las pérdidas no manifestadas por aspectos sociales que los inhabilitan en la expresión de sus emociones ${ }^{(25)}$.

Con respecto a los resultados relacionados a la unidad en la que se desempeñaban, los trabajadores de la unidad de oncología mostraron una mayor percepción de apoyo de su entorno, en comparación con los profesionales de UCI. Para algunos autores, esto puede estar dado por ciertas reglas que son compartidas por los miembros de cada unidad, las que están definidas por la estructura, el proceso interno de las prácticas diarias y del propósito ${ }^{(26,27)}$.

En consecuencia, este instrumento será de utilidad para los equipos de salud mental de los centros de atención, dado que permitirá evaluar la percepción de apoyo social de los funcionarios, considerando que varios estudios han mostrado que en el área de salud, este apoyo se genera en forma natural entre los colegas y en muy bajo número, de parte de las instituciones ${ }^{(28,29)}$. Esto adquiere relevancia considerando que el trabajo de equipo y la generación de instancias formales de apoyo en las instituciones, pueden prevenir el burnout y la "fatiga por compasión"(24). Por ello se hace imprescindible desarrollar nuevas intervenciones que sean evaluadas objetivamente, y contar con instrumentos que permitan realizar dicha medición ${ }^{(30-32)}$.

\section{REFERENCIAS BIBLIOGRÁFICAS}

1. Coca C, Arranaz P, Diéz-Asper H. Burn-out en el personal sanitario que atienten a niños al final de su vida y a sus familiares. En: Gómez Sancho M, editor. Cuidados paliativos en el niño. España: Lerko/GAFOS. 2007: 167-88.

2. Flores R. Salud, Enfermedad y Muerte: Lecturas desde la Antropología Sociocultural. Revista MAD Universidad de Chile. 2004 [Internet] [Acceso 10 de mayo de
2015]. Disponible en: http://www.rchdt. uchile.cl/index.php/RMAD/article/viewFile/14783/15122.

3. Caycedo M. La muerte en la cultura occidental: antropología de la muerte. Rev Colomb Psiquiat 2007;36:332-9.

4. Plante J, Cyr C. Health care professionals` grief after death of child. Paediatr Child Health 2011; 16:213-6. PMCID: PMC3076172

5. Folkman S. Revised coping Theory and the process of Bereavement. In: Stroebe M, Hansson O, Stroebe W, Schut H, editors. Handbook of bereavement research: Consequences, coping, and care. Washington, DC: American Psychological Association. 2003.p.563-84.

6. Grau A, Suñer R, García M. Desgaste profesional en el personal sanitario y su relación con los factores personales y ambientales. Gac Sanit 2005;19:463-70.

7. Puia D, Lewis L, Tatano C. Experiences of obstetric nurse who are present for a perinatal loss. J Obstet Gynecol Neonatal Nurs 2013; 42:321-31. Doi: 10.1111/1552

8. Doka KJ. Introducción. En Doka KJ. Disenfranchised grief: New direction, chaIlengers, and strategies for practice. Champaign, IL: Research Press. 2002: 5-22.

9. Anderson K, Gaugler J. The grief experiences of certified nursing assistant: Personal growth and complicated grief. Omega 2007;54:301-18. Doi: 10.2190/T14NW223-7612-0224

10. Grau J, Llantá MC, Massip C, Chacón M, Reyes MC, Infante O, Romero T, Barroso I, Morales D. Ansiedad y actitudes ante la muerte: revisión y caracterización en un grupo heterogéneo de profesionales que se capacita en cuidados paliativos. Pensamiento Psicológico 2008;4: 27-58.

11. Isikhan V, Comez T, Danis MZ. Job stress and coping strategies in health care professionals working with cancer patients. Eur J Oncol Nurs 2004;8:234-44. DOI:10.1016/j.ejon.2003.11.004

12. Papadatou D, Anagnostopoulos F, Monos D. Factors contributing to the development 
of burnout in oncology nursing. Br J Med Psychol 1994; 67:187-99. Doi: 10.1111/ j.2044-8341.1994.tb01785.x

13. Anderson K, Ewen $\mathrm{H}$, Miles E. The Grief support in Healthcare Scale. Nurs Res 2010; 59:372-9. Doi: 10.1097/ NNR.0b013e3181fca9de.

14. Nunnaly JC, Bernstein IH. Psychometric theory (3rd ed.). New York: McGraw-Hill, 1994.

15. Tatano C, Bernal H, Froman R. Methods to document semantic equivalence of a translated scale. RINAH. 2003; 26: 64-73. Doi: 10.1002/nur.10066

16. Hess J, Singer E. The role of respondent "debriefing" questions in questionnaire development. Proceedings of the American Statistical Association (Survey Research Methods Section), American Statistical Association, Alexandria, VA. 1995. p1075-1080.

17. Speber A. Translation and validation of study instruments for cross-cultural research. Gastroenterology 2004; 26:S124-S128. Doi: 10.1053/j.gastro.2003.10.016

18. Su, Yu-Sung, et al. Su Y-S, Gelman A, Hill J, Yajima M., Multiple imputation with diagnostics (mi) in R: Opening windows into the black box. J Stat Softw 2011; 45:1-31.

19. Husson F, Lê $S$, Pagès J. Exploratory multivariate analysis by example using R. Boca Raton: CRC press, 2010.

20. Falissard B. Psy Various procedures used in psychometry. $\mathrm{R}$ package version 1.1. 2012. [Internet] [Acceso 10 de mayo de 2015]. Disponible en: https://cran.r-project.org/web/packages/psy/psy.pdf

21. Davison A, Hinkley, DV. Bootstrap methods and their application. 1997. Cambridge: Cambridge University

22. Core R. R: A language and environment for statistical computing. 2013. Foundation for Statistical Computing, Vienna, Austria. URL http://www.R-project.org/.

23. Oviedo H, Campo A. Aproximación al uso del coeficiente alfa de Cronbach. An approach to the use of Cronbach's Alfa. Rev Colomb Psiquiatr 2005;34:572-80.
24. Meier DE, Back AL, Morrison RS. The inner life of physicians and care of seriously ill. JAMA 2001; 286:3007-14. Doi:10.1001/ jama.286.23.3007.

25. Eagle S, Creel A, Alexandrov A. The effect of facilitated peer support sessions on burnout and grief management among health care providers in pediatric intensive care units: A pilot study. J Palliat Med 2012; 15:1178-80.

26. Papadotau D. A proposed model of health profesionals'grieving process. 2000. OMEGA 2000; 41: 59-77. Doi: 10.2190/TV6M8YNA-5DYW-3C1E

27. Rashotte J, Fothergill-Bourbonnais F, Chamberlain M. Pediatric intensive care nurse and their grief experiences: A phenomenological study. Heart Lung. [Internet] 1997 [Acceso 13 de noviembre de 2011]; 26:372-86. Doi: 10.1016/S01479563(97)90024-8

28. Persaud R. Post-traumatic stress disorder in doctors. BMJ Career Focus 2005; 330:86-7.

29. Papadatou D, Martinson IM, Chung PM. Caring for dying children: a comparative study of nurses' experiences in Greece and Hong Kong. Cancer Nurs 2001; 24:402-12. Doi: 10.1097/00002820-200110000-00013

30. Keene E, Hutton N, Hall B, Rushton C. Bereavement debriefing sessions: an intervention to support health care professionals in managing their grief after the death of a patient. PN. [Internet] 2010 [acceso 23 de marzo 2011]; 36: 185-189. Disponible en: http://www.pediatricnursing.net/ ce/2012/article36185189.pdf

31. Villacieros M, Serrano I, Bermejo JC, Magaña M, Carabias R. Social support and psychological well-being as possible predictors of complicated grief in a cross-section of people in mourning. 2014. Anal Psicol 30:94451. Doi: 10.6018/analesps.30.3.154691

32. Wenzel J, Shaha M, Klimmek R, Krumm S. Working through grief and loss: oncology nurses' perspectives on professional bereavement. Oncol Nurs Forum 2011;38: E272-82. Doi: 10.1188/11.ONF.E272-E282. 


\title{
ANEXO I. ESCALA DE APOYO EN DUELO EN ATENCIÓN DE SALUD
}

Las siguientes afirmaciones se refieren al apoyo que usted recibe como profesional/ técnico de salud ante el duelo.

Por favor encierre en un círculo la respuesta que mejor describe su nivel de acuerdo o desacuerdo con cada afirmación.

\author{
TD - Totalmente en desacuerdo \\ $\mathrm{D}$ - En desacuerdo \\ $\mathrm{N}$ - Ni de acuerdo ni en desacuerdo \\ A - De acuerdo \\ TA - Totalmente de acuerdo
}

\begin{tabular}{|c|c|c|c|c|c|}
\hline Afirmación & \multicolumn{5}{|c|}{ Respuesta } \\
\hline 1. Mi familia comprende cuan cercano soy con los pacientes. & TD & $\mathrm{D}$ & $\mathrm{N}$ & A & TA \\
\hline 2. Mis amigos comprenden cuan cercano soy con los pacientes. & TD & $\mathrm{D}$ & $\mathrm{N}$ & A & TA \\
\hline $\begin{array}{l}\text { 3. Mis compañeros de trabajo comprenden cuan cercano soy con los } \\
\text { pacientes. }\end{array}$ & TD & $\mathrm{D}$ & $\mathrm{N}$ & A & TA \\
\hline 4. Mis supervisores comprenden cuan cercano soy con los pacientes. & TD & $\mathrm{D}$ & $\mathrm{N}$ & A & TA \\
\hline $\begin{array}{l}\text { 5. Los familiares de los pacientes comprenden cuan cercano soy con su } \\
\text { familiar enfermo. }\end{array}$ & TD & $\mathrm{D}$ & $\mathrm{N}$ & A & TA \\
\hline 6. Mi familia sabe que sufro cuando los pacientes fallecen. & TD & $\mathrm{D}$ & $\mathrm{N}$ & A & TA \\
\hline 7. Mis amigos saben que sufro cuando los pacientes fallecen. & TD & $\mathrm{D}$ & $\mathrm{N}$ & A & TA \\
\hline $\begin{array}{l}\text { 8. Mis compañeros de trabajo saben que sufro cuando los pacientes } \\
\text { fallecen. }\end{array}$ & TD & $\mathrm{D}$ & $\mathrm{N}$ & A & TA \\
\hline 9. Mis supervisores saben que sufro cuando los pacientes fallecen. & TD & $\mathrm{D}$ & $\mathrm{N}$ & A & TA \\
\hline $\begin{array}{l}\text { 10. Los familiares de los pacientes saben que sufro cuando su familiar } \\
\text { fallece. }\end{array}$ & TD & $\mathrm{D}$ & $\mathrm{N}$ & A & TA \\
\hline $\begin{array}{l}\text { 11. Mi institución a menudo lleva a cabo rituales en memoria de } \\
\text { pacientes que han fallecido. }\end{array}$ & TD & $\mathrm{D}$ & $\mathrm{N}$ & A & TA \\
\hline $\begin{array}{l}\text { 12. A menudo puedo asistir a los rituales en memoria que se llevan a } \\
\text { cabo en mi institución. }\end{array}$ & TD & $\mathrm{D}$ & $\mathrm{N}$ & A & TA \\
\hline $\begin{array}{l}\text { 13. A menudo soy invitado a asistir a los rituales en memoria de los } \\
\text { pacientes fallecidos, que se realizan fuera de mi institución. }\end{array}$ & TD & $\mathrm{D}$ & $\mathrm{N}$ & A & TA \\
\hline 14. A menudo puedo asistir a los funeral de pacientes que fallecen & TD & $\mathrm{D}$ & $\mathrm{N}$ & A & TA \\
\hline $\begin{array}{l}\text { 15. Mi institución me mantiene informado acerca de los fallecimientos } \\
\text { de los pacientes. }\end{array}$ & TD & $\mathrm{D}$ & $\mathrm{N}$ & A & \\
\hline
\end{tabular}

Puntaje: $\mathrm{TD}=1 ; \mathrm{D}=2 ; \mathrm{N}=3 ; \mathrm{A}=4 ; \mathrm{TA}=5$. Puntajes promedios debiesen ser calculados. Puntajes altos corresponden a altos niveles de apoyo en duelo.

Escala adaptada y validada por Vega, P. González, R. Bustos, J. Santibañez, N. Cortínez, V. Sateler, A. \& Viedma M. (2015), de la Encuesta "Grief Support Health care Scale" (GSHCS) desarrollada por Anderson, Ewen y Miles. Referencia: Anderson K, Ewen H, Miles E. The Grief support in Healthcare Scale. Nur Res. 2010; 59(6): 372-379. 